\title{
Ultrasound-guided percutaneous sclerosis of congenital splenic cysts using ethyl alcohol $96 \%$ and minocycline hydrochloride 10\%: A pediatric series
}

\author{
Antonella Accinni ${ }^{\text {a }}$, Arianna Bertocchini ${ }^{\mathrm{a}, *}$, Silvia Madafferi ${ }^{\mathrm{a}}$, Gianluigi Natali ${ }^{\mathrm{b}}$, Alessandro Inserra ${ }^{\mathrm{a}}$ \\ a General and Thoracic Surgery Unit, Department of Surgery, Bambino Gesù Children's Hospital, Rome \\ ${ }^{\mathrm{b}}$ Interventional Radiology Unit, Department of Surgery, Bambino Gesù Children's Hospital, Rome
}

\section{A R T I C L E I N F O}

\section{Article history:}

Received 11 June 2015

Received in revised form 23 May 2016

Accepted 24 May 2016

\section{Key words:}

Congenital splenic cysts

Percutaneous sclerotherapy

Minocycline hydrocloride

Ethyl alcohol

Children

\begin{abstract}
A B S T R A C T
Introduction: The management of congenital splenic cysts continues to evolve. In the past the standard treatment was splenectomy, but increased knowledge about the spleen's immunologic function has led most pediatric surgeons to preserve splenic tissue.

A great number of studies using sclerosing substances have been published, but to date reports in children have been limited. Our study concerns a group of 15 children with congenital splenic cysts treated with percutaneous drainage and sclerosis with alcohol. We performed the procedure under general anesthesia and checked radiologically for possible leakage.

Methods: In 2000 our group started managing pediatric patients with splenic cysts. During the first eight years surgery was the treatment of choice.

From April 2008 to December 2014, a prospective study was conducted on 15 consecutive patients treated with percutaneous sclerotherapy. The outcomes regarding cystic dimensional variations before and after treatment were analyzed.

Results: In $20 \%$ of patients complete disappearance of the cystic lesion was achieved. In $67 \%$ of the patients the maximum diameter of the cyst was reduced to below $50 \mathrm{~mm}$.

Conclusion: Our results should encourage the use of this treatment because it is a valid and safe option in childhood. The high success rate achieved with percutaneous drainage and sclerotherapy of cystic lesions supports our results.
\end{abstract}

(C) 2016 Elsevier Inc. All rights reserved.
Splenic cysts are rare entities, occurring in only $0.5-2 \%$ of the population in all age groups [1].

In 1829, Andral was the first to describe a splenic cyst found during autopsy, and Pean performed the first recorded splenectomy for cyst in 1867 [2].

According to the epithelial lining, Fowler, and later on Martin [3], classified non-parasitic splenic cysts (NPSC) into two categories; true cysts (primary) and pseudocysts (secondary). Primary cysts are of parasitic or non parasitic origin, while secondary cysts have usually been found to be the consequence of splenic blunt trauma.

Congenital cysts are classified as epidermoid, dermoid or endodermoid cysts [4]. The prevalence of splenic cysts is increasing, probably because of the large number of radiological examinations currently performed [5] and to the successful non-surgical management of splenic injuries. The true incidence of splenic cysts is unknown. Up to

\footnotetext{
* Corresponding author at: General and Thoracic Surgery Unit, Department of Surgery, Bambino Gesù Children's Hospital, Piazza Sant'Onofrio 4, 00165 Roma, Italy. Tel.: + 39 3383203 451, + 390668592 423; fax: + 390668592876 .

E-mail addresses: arianna.bertocchini@opbg.net, arianna_bertocchini@hotmail.com (A. Bertocchini).
}

now, no evidence-based management has been described [6]. Primary splenic cysts represent $25 \%$ of the total number [2] and are more frequently observed in children and young adults [7]. Most cysts are asymptomatic and incidentally diagnosed during abdominal imaging or physical examination of the abdomen.

Also, some patients present symptoms related to complications of the cysts (compression, hemorrhage, rupture and infection) [8]. Treatment is recommended for symptomatic and bigger lesions. The management of congenital splenic cysts continues to evolve. In the past splenectomy was the standard treatment, but during the last two decades, various preservation procedures have become the preferred approach of most pediatric surgeons [9]. However, the recurrence rate may be high [10], and such procedures have now been abandoned by many groups and new interventional radiological approaches are starting to be tried [11].

In this report we propose a conservative, spleen preserving therapy for splenic cysts using ultrasonography-guided fine needle aspiration and subsequent sclerotherapy with ethyl alcohol $96 \% \mathrm{v} / \mathrm{v}$ sterile and minocycline hydrochloride $10 \%$.

Despite the complications associated with treatment by percutaneous drainage and injection of a sclerosing agent, as well as the 
significant recurrence rate described in literature [10], our team preferred this approach which preserves the spleen. This report presents a prospective series of 15 cases of pediatric splenic cysts treated with a sclerosing agent, reviews the current status of pediatric splenic cysts in literature, and analyzes the current classification and treatment methods.

\section{Patients and methods}

The institutional scientific board approved this study.

Based on results previously obtained for 11 patients treated in our unit with other invasive methods, in April 2008 we started a prospective study on 15 consecutive patients with splenic cysts treated by percutaneous sclerotherapy in a single center. Inclusion criteria were presence of symptoms or a cyst diameter of $50 \mathrm{~mm}$ or more. The clinical, radiological and cytological findings were analyzed. The data collected concerned age, sex, previous history of trauma, presenting symptoms and physical examination findings, radiological findings, type of management and outcome. Laboratory assessment included a standard preoperative blood sample and Echinococcus antibody dosage. Radiological scheduled assessments included only ultrasound (US) evaluation. Written informed consent was obtained from parents of all patients before the interventional procedure.

Minocycline hydrochloride also was used by Shimanuki in 1996 [12] as a successful sclerosing agent for the eradication of an epidermoid splenic cyst. Minocycline hydrochloride is an antibiotic known to have a particular sclerosing effect. We decided to use this agent in association with alcohol for the treatment of splenic cysts because it can be left inside the cysts without any risk of chemical damage.

The sclerosis was performed under general anesthesia. Local anesthesia with lidocaine hydrochloride was used only in one 17-year-old patient. The procedure was performed by one experienced interventional radiologist in a standardized manner.

The cyst was punctured directly, in sterile conditions, under ultrasound guidance, and respecting the splenic tissue. After complete suction of the cystic contents, a fluoroscopic cystography (Fig. 1) was performed to detect any leakage around the catheter, into the peritoneum and any small communicating vessel draining into the splenic vein. An adequate amount (30-35\% of the volume of the cyst) of a sclerosing agent (ethyl alcohol 96\%) was then injected.

The alcohol was left inside the cavity for $30 \mathrm{~min}$.

Since the first report by Akhan in 1997 [11], alcohol has been widely used for splenic cysts with high success rates [11,13], while other agents have been shown to have major disadvantages: high failure rates, high toxicity and recurrences were reported $[14,15]$. The alcohol leads to fixation of the cells lining the cyst cavity thus preventing fluid secretion.

Since the failure of alcohol to cover the complete surface of the cyst wall may result in persistence of some cells on the cyst wall with persistent fluid secretion and cyst recurrence [16], we followed the suggestion of Akhan et al. [11] to roll the patients to the left and to the right after the alcohol injection and left in each position for some minutes to permit the alcohol to act, ensuring its fixation and cessation of cyst fluid secretion. After the procedure, all the sclerosing substance was drawn and a 6 Fr pigtail drain was left inside the lesion.

After $24 \mathrm{~h}$ a new bolus of ethyl alcohol $96 \%$ was injected. Thirty minutes later the sclerosing agent was replaced with $10 \mathrm{ml}$ of minocycline hydrochloride $10 \%$. The drain was then removed leaving the antibiotic in the cavity.

The patient was hospitalized post-procedure for observation, monitoring of fever and pain, and standardized antibiotic and analgesic therapy. The procedure was repeated if the lesion persisted.

Outcome with regard to cystic dimensional variation after treatment was analyzed with ultrasonography every three months in the first year, every six months during following 2 years and once a year thereafter.

All numeric data are presented as mean and standard deviation. Range and median value of follow-up period after last sclerosis are reported.

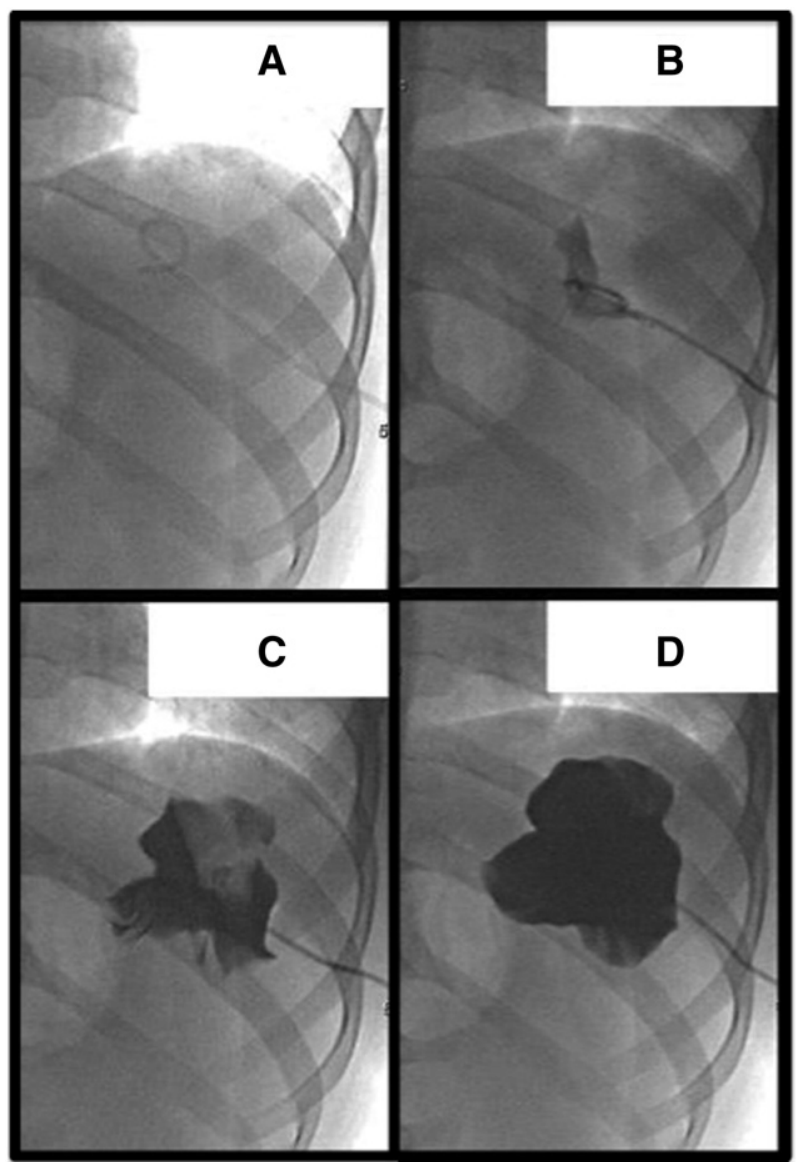

Fig. 1. Sequence of consecutive images during fluoroscopic cystography check routinely performed before alcohol injection. (A) View of pig tail. (B, C) Contrast injection into the cyst. (D) Complete opacification of the whole cyst without leakage.

\section{Results}

All data concerning the population studied are summarized in Table 1.

Mean age at diagnosis was 12.54 years and SD was 4.18. There were eight males and seven females. Only one child had a history of antecedent trauma (C).

In six patients the presenting symptom was left upper quadrant discomfort (A, C, E, K, L, O) and feeling of fullness in one (D). No anamnestic data about symptoms of patient $B$ were registered. The remaining patients (F, G, H, I, J, L, M, N) were asymptomatic even though two (F, $\mathrm{G}$ ) presented a big abdominal mass. Patients HIJLMN came to our knowledge and to radiologic imaging, by chance in an examination for another condition. They all were patients otherwise healthy and they did not have any underlying diseases or infections in the past.

On physical examination, six patients (A, B, C, D, F, G) had a left upper quadrant large palpable abdominal mass, but the other nine (E, $\mathrm{H}, \mathrm{I}, \mathrm{J}, \mathrm{K}, \mathrm{L}, \mathrm{M}, \mathrm{N}, \mathrm{O}$ ) had no palpable lesions. All patients underwent radiological assessment. In particular, all lesions were clearly studied by US revealing a well-marginated homogeneous anechoic area with floating, small echo enhancements, thin septations and smooth thin wall.

All patients underwent US by our radiologists, in addition patients ABCLO underwent CT scan and patients DGHM MRI, performed elsewhere (Fig. 2). Laboratory data were unremarkable in all cases and all patients were negative for echinococcus antibodies. No bacterial infections were detected. At diagnosis the mean maximum diameter of the cysts was $94 \mathrm{~mm}$.

In 11 patients a single sclerosing session was performed ( $B, C, E, H, I$, J, K, L, M, N, O); ethyl alcohol 96\% and minocycline hydrochloride $10 \%$ 
Table 1

Summary of clinical, radiological features and follow up of patients with congenital splenic cysts treated with percutaneous sclerosis.

\begin{tabular}{|c|c|c|c|c|c|c|c|c|c|c|c|}
\hline & Age & Sex & Symptoms & $\begin{array}{l}\text { Physical } \\
\text { examination }\end{array}$ & Imaging & $\begin{array}{l}\text { Maximum cyst } \\
\text { diameter at } \\
\text { diagnosys }(\mathrm{mm})\end{array}$ & $\begin{array}{l}\text { Maximum cyst } \\
\text { diameter after } \\
\text { treatment }(\mathrm{mm})\end{array}$ & $\begin{array}{l}\text { Percentage of } \\
\text { maximum } \\
\text { diameter reduction }\end{array}$ & $\begin{array}{l}\text { Number of } \\
\text { treatments }\end{array}$ & Complications & $\begin{array}{l}\text { Follow up } \\
\text { (mo) }\end{array}$ \\
\hline A & 8.92 & $\mathrm{~F}$ & $\mathrm{AP}$ & PM & US, CT & 100 & 0 & $100 \%$ & 2 & None & 79 \\
\hline $\mathrm{B}^{*}$ & 12.75 & M & $(* *)$ & PM & US, CT & 99 & $26-27$ & $(* * *)$ & 1 & $\begin{array}{l}\text { AP } \\
\text { IPFC }\end{array}$ & 77 \\
\hline $\mathrm{C}$ & 5.08 & M & $\mathrm{AP}$ & PM & US, CT & 30 & 0 & $100 \%$ & 1 & None & 69 \\
\hline $\mathrm{D}$ & 17.2 & $\mathrm{~F}$ & SF & PM & US, MRI & 200 & 78 & $61 \%$ & 4 & None & 47 \\
\hline $\mathrm{E}$ & 3.4 & $\mathrm{~F}$ & $\mathrm{AP}$ & None & US & 38 & 0 & $100 \%$ & 1 & None & 59 \\
\hline $\mathrm{F}$ & 13.67 & $\mathrm{~F}$ & None & PM & US & 150 & 34 & $77.3 \%$ & 2 & None & 59 \\
\hline G & 16.5 & M & None & PM & US, MRI & 230 & 30 & $86.9 \%$ & 2 & None & 51 \\
\hline $\mathrm{H}$ & 17.4 & $\mathrm{~F}$ & None & None & US, MRI & 45 & 15 & $66.6 \%$ & 1 & None & 30 \\
\hline I & 14.8 & M & None & None & US & 78 & 20 & $74.3 \%$ & 1 & None & 17 \\
\hline $\mathrm{J}$ & 13.4 & M & None & None & US & 130 & 48 & $63.1 \%$ & 1 & None & 17 \\
\hline $\mathrm{K}$ & 11.9 & $\mathrm{~F}$ & $\mathrm{AP}$ & None & US & 56 & 7 & $87.5 \%$ & 1 & None & 17 \\
\hline $\mathrm{L}$ & 17.4 & M & $\mathrm{AP}$ & None & US, CT & 74 & 22 & $70.2 \%$ & 1 & None & 15 \\
\hline M & 11.6 & M & None & None & US, MRI & 50 & 20 & $60 \%$ & 1 & None & 5 \\
\hline $\mathrm{N}$ & 12.2 & $\mathrm{~F}$ & None & None & US & 50 & 24 & $52 \%$ & 1 & None & 5 \\
\hline 0 & 11.7 & $\mathrm{M}$ & $\mathrm{AP}$ & None & US, CT & 80 & 62 & $22.5 \%$ & 1 & None & 3 \\
\hline
\end{tabular}

injection was repeated twice in three patients $(A, F, G)$ and four times in one (D).

In all patients the procedure was done under general anesthesia. In one patient (D) sclerosis was started under local anesthesia with $2 \mathrm{mg} / \mathrm{kg} /$ dose of lidocaine hydrochloride but was immediately suspended because of excessive pain then resumed under general anesthesia. Aspirated fluids were clear or slightly turbid in all procedures. In all patients the cytopathological examination was negative for malignant cells and revealed no cellular atypia; there were only debris, scant cells, lymphocytes and leukocytes. Twenty-four hours later, all patients were given a local injection of $10 \mathrm{ml}$ of minocycline hydrochloride $10 \%$.

No procedural complications were observed. No toxicity effect occurred.

In post-operative period all patients were given amoxicillin and clavulanic acid ( $50 \mathrm{mg} / \mathrm{kg} /$ day) twice a day for seven days and paracetamol (10-15 mg/kg/dose) every 6-8 hours if necessary.

A post-procedural early complication occurred in a single patient (B), with abdominal pain onset registered on the first post-operative day. US check revealed a $12-\mathrm{mm}$ intraperitoneal fluid collection in the rectovesical pouch. Abdominal pain, treated with intravenous injection of paracetamol, resolved on the third post-operative day and fluid collection disappeared at US control performed on the fourth postoperative day. The patient was discharged in good condition on the fifth post-operative day. Neither secondary infection nor fever occurred in any patient.

The median value of hospitalization after first treatment was 2 (range 1-5) days, 2 (range 1-2) days after second treatment, 1 day after the third and fourth treatments. In three patients the cysts completely disappeared, after one treatment (C, E) and after two treatments (A). In ten cases (patient B, F, G, H, I, J, K, L, M, N) sclerosis determined a considerable reduction in cyst dimensions. In patient B the US follow up showed a partition of residual cyst into two smaller lesions with a maximum diameter of $27 \mathrm{~mm}$ and $26 \mathrm{~mm}$, respectively (Fig. 3). In one patient there was no significant reduction in the size of the cysts $(22.5 \%)$ but the follow-up was rather short (O) (only three months).

In one case (D) the 4 consecutive sessions determined an important but insufficient reduction of the cyst whose maximum diameter fell from $200 \mathrm{~mm}$ to $78 \mathrm{~mm}$. Further sclerosis was proposed but refused for teenage rebellion of the patient. The patient continued with US follow up and the cyst size has remained stable.

The median length of follow up was 30 months (range 3-79 months) months) including the patient that abandoned treatment after only 3 months. No late complications were observed. After treatment the seven symptomatic patients became asymptomatic and no mass was palpable at physical examination in any patient.

\section{Discussion}

Until now, the standard treatment for congenital splenic cysts has been an open or laparoscopic surgery with partial or total splenectomy.

Recently a spleen sparing approach has been proposed and preferred especially for the pediatric population [17]. Its purpose is to preserve splenic immune function and avoid overwhelming postsplenectomy infection (post-splenectomy sepsis has a reported associated mortality rate of $1.5 \%$ [18]).

The aim of our study is to propose the US-guided percutaneous sclerotherapy as a minimally invasive and spleen-sparing alternative

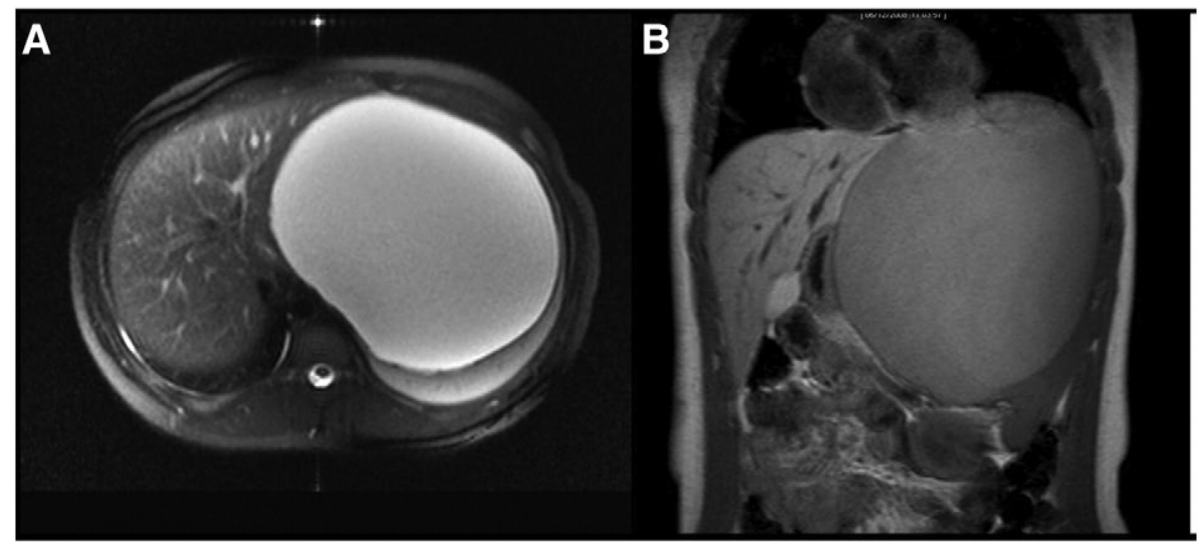

Fig. 2. RM transversal (A) and coronal (B) views of the splenic lesion in patient D. 


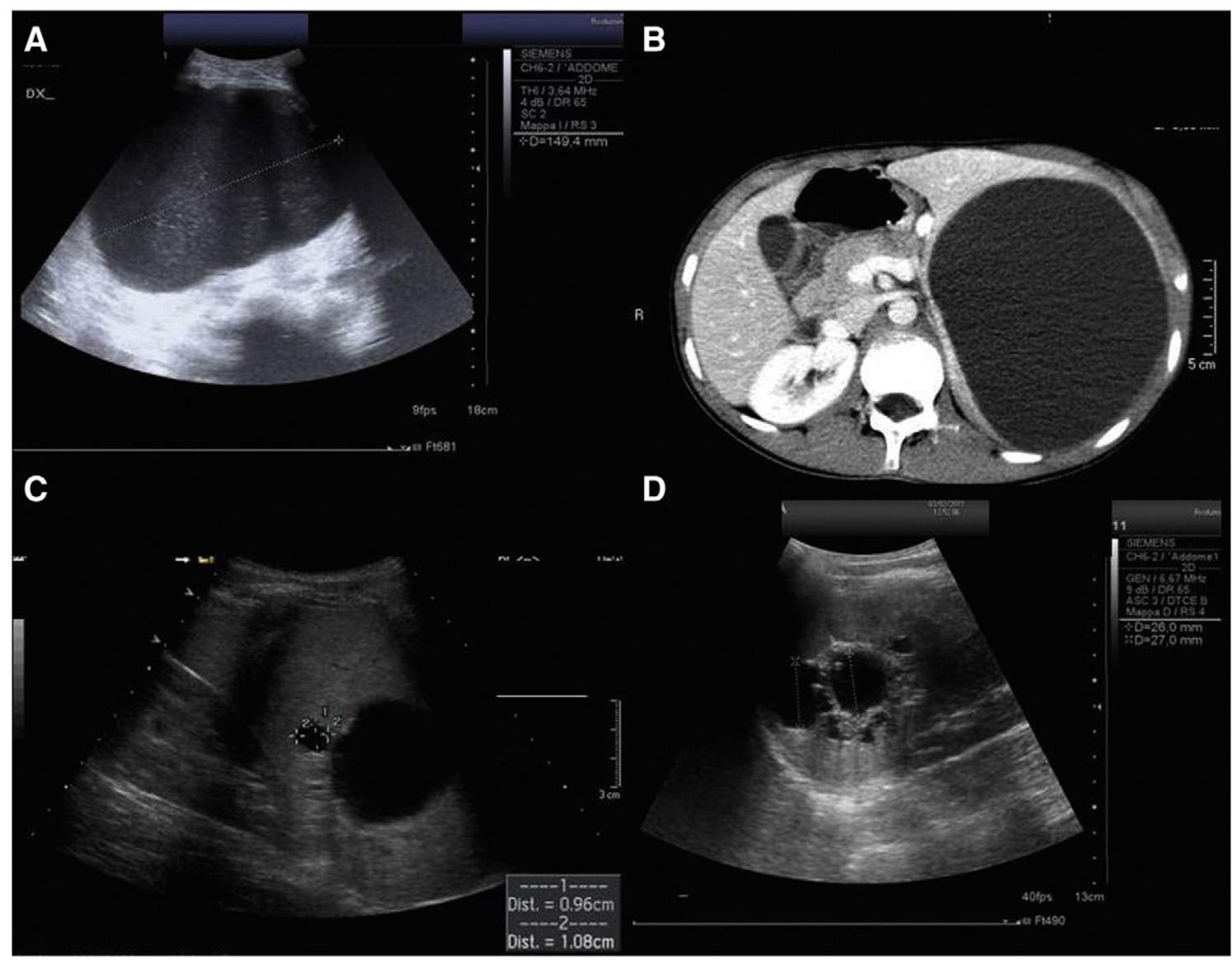

Fig. 3. Patient B US image (A) and CT view (B) at diagnosis. Partial reduction and partition into two cysts during treatment course (C). Further reduction at the end of therapy (D).

approach to traditional treatments for children affected by large or symptomatic splenic cysts.

According to literature, we considered our technique effective when the maximum diameter was reduced to below $50 \mathrm{~mm}$. With our procedure overall treatment efficacy was good: in $20 \%$ of patients the cystic lesion completely disappeared, in $67 \%$ the maximum diameter decreased to less than $50 \mathrm{~mm}$ and symptoms disappeared and no patient underwent surgical treatment.

Even if in 2010 Boybeyi [8] advised against the use of percutaneous aspiration and sclerosis because he had observed relapse after this procedure, in our study, there were no recurrences or postoperative complications, and all patients but one is currently asymptomatic and cysts are smaller than $50 \mathrm{~mm}$.

A wide range of spleen-preserving treatment modalities has been described in literature for symptomatic or very large splenic cysts as cyst aspiration, marsupialization (internal/external), partial cystectomy (decapsulation/deroofing) and total cystectomy [9,19]. These preserving surgical approaches have been abandoned by most surgeons because of the high recurrence rate of the splenic cysts (Table 2), as

Table 2

Review of operative procedures and relative recurrence rates published by Sinha

\begin{tabular}{lcc}
\hline Procedure & Number (Total 157) & Recurrence \\
\hline Open total splenectomy & $11 \%(\mathrm{n}=18)$ & $0 \%(0 / 18)$ \\
Laparoscopic total splenectomy & $2 \%(\mathrm{n}=3)$ & $0 \%(0 / 3)$ \\
Open partial splenectomy & $29 \%(\mathrm{n}=45)$ & $2 \%(1 / 45)$ \\
Laparoscopic partial splenectomy & $4 \%(\mathrm{n}=7)$ & $0 \%(0 / 7)$ \\
Open cystectomy & $20 \%(\mathrm{n}=31)$ & $3 \%(1 / 31)$ \\
Laparoscopic cystectomy & $10 \%(\mathrm{n}=16)$ & $50 \%(8 / 16)$ \\
Laparoscopic unroofing/decapsulation & $24 \%(\mathrm{n}=37)$ & $49 \%(18 / 37)$ \\
\hline
\end{tabular}

described by Schier in 2007 and Sinha in 2011 [17,20], and to the complications described in literature [21].

Sinah, in particular, presented a systematic study on 157 nonparasitic splenic cysts (NPSC) treated with various surgical procedures [17]. This review found that the recurrence rate with the laparoscopic partial and total cystectomy was unacceptably high (respectively 50\% and $49 \%$ ). The latter could probably be attributed to the demanding technique of peeling the cyst wall off laparoscopically.

Other authors report that recurrence rates after laparoscopic splenic cyst excision range from $50 \%$ to $71 \%$ and may depend on the type of laparoscopic resection performed (ie, unroofing vs partial splenectomy) $[15,22]$.

Small pediatric case series have reported postlaparoscopy recurrence rates as high as 71\% [16] but are insufficiently powered to determine whether specific types of laparoscopic resections are associated with higher rates of recurrence. There is some early evidence that aggressive partial splenectomy instead of simple unroofing can decrease the post-laparoscopy recurrence rate in children [15,16,22].

However, total cystectomy with open approach has a low recurrence rate, and hence proved to be an acceptable option. Also partial splenectomy (either laparoscopic or open depending on surgeon's expertise) would be a safe option with almost similar outcome. Recently new conservative interventional radiology approaches have been encouraged. Percutaneous aspiration without sclerosis has been reported to be a successful treatment, but most investigators agree that simple aspiration of the cyst does not prevent recurrences, probably because of the persistence of a true secreting epithelial lining of the cyst $[11,13,23]$. For this reason injection of chemical agents, after aspiration, has been explored as a good alternative in the attempt to collapse the cyst wall, promote fibrosis and prevent re-accumulation [24]. Alcohol [11], formalin [25], phenol [26], pantopaque [27], doxycycline or tetracycline [23] have 
been used as sclerosing agents for percutaneous procedures of different abdominal fluid lesions. No success has been reported for tetracycline injection [28]. In literature there are many reports about sclerotic treatment with alcohol in adults with good results [11,12,13,29,30], but few cases in pediatric age $[23,29,31,32]$.

On the base of these encouraging data, we decided to treat all congenital splenic cysts larger than $50 \mathrm{~mm}$ or symptomatic with alcohol sclerotherapy as first procedure. Spleen sparing surgery would be considered as a second option only in the event of failure of sclerosing management, e.g., persistence of symptoms, maximum spleen diameter greater than $50 \mathrm{~mm}$ after treatment, or persistence of palpable masses.

We believe that this procedure must be performed only by skilled interventional radiologists, alcohol being a toxic agent that can cause immediate vessel sclerosis and occlusion if injected intravascularly. The intraoperative use of US and fluoroscopy can provide an effective tool to avoid complications such as chemical peritonitis, sclerosis of vessels or portal vein thrombosis.

The number of patients is unfortunately too few to provide statistically significant results but the high success rate achieved with percutaneous drainage and sclerotherapy with alcohol in renal cysts, hepatic cysts and lymphoceles, as well as the promising report of Akhan et al., supports our results $[7,8,24-26,30]$.

\section{Conclusion}

This prospective study shows that percutaneous sclerosing treatment for splenic cysts is safe, easy and useful and our results should encourage its use in pediatric patients. It is an effective alternative therapy which preserves a sufficient amount of splenic parenchyma, is minimally invasive, avoids the sequelae of laparotomy and splenectomy and reduces the duration of postoperative hospitalization.

We believe that treatment of splenic cysts with percutaneous drainage and sclerosis with alcohol is a valid option and does not rule out surgery if the conservative treatment fails.

\section{Conflict of interest}

None of the authors have any significant disclosure related to this manuscript or its publication.

\section{References}

[1] Higaki K, Jimi A, Watanabe J, et al. Epidermoid cyst of the spleen with CA19-9 or carcinoembryonic antigen productions: Report of three cases. Am J Surg Pathol 1998;22(6):704-8.

[2] Andral G. Precis d'anatomie pathologique. Paris: Gabon; 1829432.
[3] Martin JW. Congenital splenic cysts. Am J Surg 1958;96(2):302-8.

[4] Reddi VR, Reddy MK, Srinivas B, et al. Mesothelial splenic cyst-a case report. Ann Acad Med Singapore 1998;27(6):880-2.

[5] Robertson F, Leander P, Ekberg O. Radiology of the spleen. Eur Radiol 2001;11(1): 80-95.

[6] Geraghty M, Khan IZ, Conlon KC. Large primary splenic cyst: A laparoscopic technique. J Minim Access Surg 2009;5(1):14-6.

[7] Miele V, Galluzzo M, Cortese A, et al. Diagnostic imaging of splenic cysts in children. Radiol Med 1998;95(1-2):62-5.

[8] Boybeyi O, Karnak I, Tanyel FC, et al. The management of primary nonparasitic splenic cysts. Turk J Pediatr 2010;52(5):500-4.

[9] Morgenstern L. Nonparasitic splenic cysts: Pathogenesis, classification, and treatment. J Am Coll Surg 2002;194(3):306-14.

[10] Mahomed A, Merry C, Guiney EJ. Splenic cysts - aspiration or partial splenic decapsulation? S Afr J Surg 1998;36(3):84-6.

[11] Akhan O, Baykan Z, Oguzkurt L, et al. Percutaneous treatment of a congenital splenic cyst with alcohol: A new therapeutic approach. Eur Radiol 1997;7(7):1067-70.

[12] Shimanuki K, Satake M. Non surgical treatment of splenic cyst, using with installation of minocycline chloride. Fukushima J Med Sci 1996;42(1-2):23-30.

[13] Volk M, Rogler G, Strotzer M, et al. Post-traumatic pseudocyst of the spleen: Sclerotherapy with ethanol. Cardiovasc Intervent Radiol 1999;22(3):246-8.

[14] Khelif K, Maassarani F, Dassonville M, et al. Laparoscopic partial splenectomy using radiofrequency ablation for non-parasitic splenic cysts in two children. J Laparoendosc Adv Surg Tech A 2006;16(4):414-7.

[15] Mertens J, Penninckx F, DeWever I, et al. Long-term outcome after surgical treatment of nonparasitic splenic cysts. Surg Endosc 2007;21(2):206-8.

[16] Bean WJ, Rodan B. Hepatic cyst: Treatment with alcohol. Am J Radiol 1985;144(2): 237-41.

[17] Sinha CK, Agrawal M. Non parasitic splenic cysts in children: Current status. Surgeon 2011;9(1):49-53.

[18] Kimber C, Spitz L, Drake D, et al. Elective partial splenectomy in childhood. J Pediatr Surg 1998;33(6):826-9.

[19] Hansen MB, Moller AC. Splenic cysts. Surg Laparosc Endosc Percutan Tech 2004; 14(6):316-22.

[20] Schier F, Waag KL, Ure B. Laparoscopic unroofing of splenic cysts results in a high rate of recurrences. J Pediatr Surg 2007;42:1860-3.

[21] Kenney CD, Hoeger YE, Yetasook AK, et al. Menagement of non-parasitic splenic cysts: Does size really matter? J Gastrointest Surg 2014;18(9):1658-63.

[22] Czauderna P, Vajda P, Schaarschmidt K, et al. Nonparasitic splenic cysts in children: a multicentric study. Eur J Pediatr Surg 2006;16:415-9.

[23] Jequier S, Guttman F, Lafortune M. Non-surgical treatment of a congenital splenic cyst. Pediatr Radiol 1987;17(3):248-9.

[24] Amr AE. Splenic cysts, many questions are yet to be answered: A case report. Cases J 2009;2:8474.

[25] Rosemberg GV. Solitary non-parasitic cyst of the liver. Am J Surg 1956;91(3):441-4

[26] Pearman RO. Percutaneous needle puncture and aspiration of renal cysts: A diagnostic and therapeutic procedure. J Urol 1966;96:139-45.

[27] Goldstein HM, Carlyle DR, Nelson RS. Treatment of symptomatic hepatic cyst by percutaneous installation of Pantopaque. AJR Am J Roentgenol 1976;127(5):850-3.

[28] Moir C, Guttman F, Jequier S, et al. Splenic cysts: Aspiration, sclerosis or resection. J Pediatr Surg 1989;24(7):646-8.

[29] Goktay AY, Secil M, Ozcan MA, et al. Percutaneous treatment of congenital splenic cysts: Drainage and sclerotherapy with polidocanol. Cardiovasc Intervent Radiol 2006;29(3):469-72.

[30] Thanos L, Mylona S, Ntai S, et al. Percutaneous treatment of true splenic cyst: Report of two cases. Abdom Imaging 2005;30(6):773-6.

[31] Añon R, Guijarro J, Amoros C, et al. Congenital splenic cyst treated with percutaneous sclerosis using alcohol. Cardiovasc Intervent Radiol 2006;29(4):691-3.

[32] De Caluwé D, Phelan E, Puri P. Pure alcohol injection of a congenital splenic cyst: A valid alternative? J Pediatr Surg 2003;38(4):629-32. 